\title{
Aceitabilidade sensorial do iogurte tipo grego com adição de doce de buriti (Mauritia
}

\author{
flexuosa L.) \\ Sensory acceptability of greek yogurt with buriti (Mauritia flexuosa L.) marmalade added \\ Aceptabilidad sensorial del yogur tipo griego con dulce buriti añadido (Mauritia flexuosa $\mathbf{L}$.)
}

Recebido: 23/09/2021 | Revisado: 29/09/2021 | Aceito: 02/10/2021 | Publicado: 04/10/2021

\author{
Natália Lira Barboza \\ ORCID: https://orcid.org/0000-0002-3249-3570 \\ Universidade Federal do Amazonas, Brasil \\ E-mail: natalia.lira83@gmail.com \\ Erika Santiago da Silva \\ ORCID: https://orcid.org/0000-0001-9191-8324 \\ Universidade Federal do Amazonas, Brasil \\ E-mail: erikasan.k@gmail.com \\ Joyce Paixão do Nascimento \\ ORCID: https://orcid.org/0000-0001-9774-6041 \\ Universidade Federal do Amazonas, Brasil \\ E-mail: joycepaixaon@gmail.com \\ Maria Clara Machado Lemos \\ ORCID: https://orcid.org/0000-0002-6764-5845 \\ Universidade Federal do Amazonas, Brasil \\ E-mail: claaralemos@outlook.com \\ Yanka Silva Vasconcelos \\ ORCID: https://orcid.org/0000-0002-2086-2703 \\ Universidade Federal do Amazonas, Brasil \\ E-mail: yankasv17@gmail.com \\ Eyde Cristianne Saraiva Bonatto \\ ORCID: https://orcid.org/0000-0002-5553-0127 \\ Universidade Federal do Amazonas, Brasil \\ E-mail: eydecristianne@gmail.com
}

\begin{abstract}
Resumo
O buriti (Mauritia flexuosa L.) é uma fruta nativa da Região Amazônica, fonte de vitaminas A, C e E e nutrientes importantes para a nutrição humana. A inserção da polpa da fruta em iogurtes surge como alternativa funcional e benéfica, de alto valor agregado e importância sociocultural. Dessa forma, o objetivo deste trabalho foi desenvolver um iogurte tipo grego com adição de doce de buriti e avaliar sua aceitabilidade sensorial. O iogurte grego foi preparado com leite UHT desnatado no Laboratório de Bioenergia, da Universidade Federal do Amazonas. Para testar a aceitabilidade, foi realizada uma análise sensorial com 50 provadores não treinados, onde foi aplicada uma ficha de avalição com uma escala hedônica de nove pontos com os extremos "gostei extremamente" e "desgostei extremamente", submetidos para uma avaliação de atributos sensoriais (aroma, aparência, cor, sabor e textura). O atributo sabor obteve a maior nota média $(8,08)$, seguido pela cor $(7,54)$, a textura $(7,42)$, o aroma $(6,94)$ e a aparência $(6,9)$. O produto elaborado mostrou ser um potencial competitivo em comparação a outros iogurtes gregos produzidos com doces de frutas amazônicas e frutas convencionais, sendo fortemente recomendado como uma ideia inovadora no mercado de iogurtes com identidade regional.
\end{abstract}

Palavras-chave: Aceitabilidade sensorial; Buriti; Iogurte grego.

\begin{abstract}
Buriti (Mauritia flexuosa L.) is a native fruit from the Amazon region, source of vitamins A, C and E and important nutrients for human nutrition. The addition of fruit pulp in yogurts appears as a functional and beneficial alternative, with high added value and sociocultural significance. Thus, the aim of this study was to develop a Greek type yogurt with buriti marmalade added and evaluate its sensory acceptability. Greek yogurt was produced with skimmed UHT milk in the Bioenergy Laboratory at Federal University of Amazonas. To test acceptability, a sensory analysis was performed with 50 untrained tasters, where an evaluation form with a nine-point hedonic scale was applied, ranging from "like extremely" to "dislike extremely", set for an evaluation of sensory attributes (aroma, appearance, color, flavor and texture). The attribute flavor obtained the highest average score (8.08), followed by color (7.54), texture (7.42), aroma (6.94) and appearance (6.9). The elaborated product shown to have a competitive potential compared to other Greek yogurts obtained with Amazonian and conventional fruits marmalades, being strongly recommended as an innovative idea in the yogurt market with regional identity.
\end{abstract}

Keywords: Sensory acceptability; Buriti; Greek yogurt. 


\section{Resumen}

Buriti (Mauritia flexuosa L.) es una fruta nativa de la región amazónica, fuente de vitaminas A, C y E y nutrientes importantes para la nutrición humana. La inclusión de pulpa de fruta en yogures aparece como una alternativa funcional y beneficiosa, de alto valor agregado e importancia sociocultural. Así, el objetivo de este trabajo fue desarrollar un yogur tipo griego con el agregado de mermelada de burití y evaluar su aceptabilidad sensorial. El yogur griego se preparó con leche desnatada UHT en el Laboratorio de Bioenergía de la Universidad Federal de Amazonas. Para probar la aceptabilidad, se llevó a cabo un análisis sensorial con 50 catadores no entrenados, donde se aplicó un formulario de evaluación con una escala hedónica de nueve puntos con los extremos "me gustó mucho" y "no me gustó mucho", sometido para una evaluación de atributos sensoriales (aroma, apariencia, color, sabor y textura). El atributo sabor tuvo el puntaje promedio más alto (8.08), seguido del color (7.54), textura (7.42), aroma (6.94) y apariencia (6.9). El producto elaborado demostró tener un potencial competitivo frente a otros yogures griegos elaborados con mermeladas de frutas amazónicas y frutas convencionales, siendo muy recomendable como idea innovadora en el mercado del yogur con identidad regional.
\end{abstract}

Palabras clave: Aceptabilidad sensorial; Burití; Yogur griego.

\title{
1. Introdução
}

Na Amazônia existem inúmeras espécies vegetais com potencial econômico, entre elas destaca-se o buruti (Mauiritia flexuosa L.), desempenhando um papel importante na fauna, uma vez que seus frutos servem de alimento para muitas espécies de pássaros e mamíferos (Vieira, et al., 2010). O fruto possui uma polpa de coloração amarelo alaranjada, com sabor agridoce e consistência amilácea e oleosa (Beltrão \& Oliveira, 2007), tendo praticamente todas as suas partes aproveitáveis, incluindo o tronco do qual se extrai a fécula e o palmito (Loureiro et. al, 2013).

De acordo com Aguiar e Souza (2017), a polpa do buriti apresenta quantidades consideráveis de carotenoides, polifenóis e ácido ascórbico. Estudos recentes têm demonstrado efeitos benéficos do consumo da fruta, como a ingestão de vitaminas A, C e E (Oliveira e Silva, et al., 2012; Gao, et al., 2012; Wang, et al., 2012). Devido ao seu sabor acentuado e acidez considerável, o seu consumo torna-se limitado a sucos e néctares, por isso, como alternativa, sugere-se a inclusão da fruta no desenvolvimento de novos produtos que permitam manter suas propriedades funcionais e nutricionais, agregando valor ao buriti e gerando uma opção de atividade sustentável para populações da região (Castro, et al., 2014). A inserção da polpa da fruta em iogurtes surge como sugestão.

Para Kailasapathy, et al. (2010), o consumo de produtos alimentícios com altos teores funcionais têm ganhado espaço no mercado, o iogurte supre a necessidade de um produto completo, aqueles desenvolvidos com adição de frutas se tornam uma alternativa viável para a indústria de alimentos, uma vez que possuem ótima aceitação pelos consumidores.

De acordo a Instrução Normativa $\mathrm{n}^{\circ}$ 46, de 23 de outubro de 2007, entende-se como iogurte o produto “cuja fermentação se realiza com cultivos protosimbióticos de Streptococcus salivarius subsp. thermophilus e Lactobacillus delbrueckii subsp. Bulgaricus, aos quais se podem acompanhar, de forma complementar, outras bactérias ácido-lácticas que, por sua atividade, contribuem para a determinação das características do produto final” (Brasil, 2007).

Os iogurtes mais concentrados apresentam maior destaque em todo mundo, com diferentes nomes de acordo com a região, labneh (leste), skyr (Islândia), shrikhand (Índia) e iogurte grego (Aryana \& Also, 2017; Uduwerella, et al., 2018). Esse último apresenta uma ótima aceitação, alto valor comercial, maior firmeza e viscosidade devido ao seu processo tecnológico de drenagem, onde há uma concentração de proteínas e gorduras (Bezerra, et al., 2019).

Considerando que o buriti é uma fruta com vários benefícios para a saúde e, quando adicionado ao iogurte grego, gera mais uma opção no mercado alimentício para o consumidor, o objetivo do trabalho foi testar a aceitabilidade do iogurte tipo grego com adição de doce de buriti. 


\section{Metodologia}

\subsection{Elaboração do iogurte grego}

A pesquisa teve caráter descritivo com método de análise qualitativo (Pereira, et al., 2018). A elaboração do iogurte grego foi realizada no Laboratório de Bioenergia, da Universidade Federal do Amazonas (UFAM), em 2017. Utilizou-se o leite UHT desnatado e a quantidade utilizada de leite, leite em pó e açúcar refinado foi adaptada de Azoubel \& Barros e Silva (2007), onde para cada 2 litros de leite foi adicionado $6 \%$ de leite em pó $(60 \mathrm{~g})$ e $32 \%$ de açúcar refinado (320g) para a correção dos teores de sólidos totais.

Após uma rápida homogeneização do leite com os ingredientes foi feito o processo de pasteurização, conforme descrito por Gomes, et al. (2016), a temperatura foi de $83{ }^{\circ} \mathrm{C}$ por trinta minutos em banho-maria digital, um litro por vez. Em seguida foi adicionado o fermento lácteo e resfriado para temperatura de $45{ }^{\circ} \mathrm{C}$ em incubadora B.O.D. O processo de coagulação foi de 6 horas. Após a coagulação a coalhada foi transferida para o dessorador de iogurte com o filtro de papel, a fim de realizar a retirada do soro, e colocado sob refrigeração à temperatura de $5{ }^{\circ} \mathrm{C}$ por 6 horas. Após esse período, a massa foi retirada e colocada em potes de plástico.

\subsection{Elaboração do doce de buriti}

Houve também a preparação do doce de buriti no Laboratório de Origem Vegetal, na Universidade Federal do Amazonas. Onde consistiu, para 500g de polpa de buriti foram adicionados 0,0625g de amido de milho, $240 \mathrm{~g}$ de açúcar e 1000 $\mathrm{mL}$ de água, homogeneizados em uma panela a fogo baixo até atingir consistência, logo após resfriado e adicionado ao iogurte grego.

\subsection{Análise sensorial}

A análise foi realizada no Laboratório de Análise Sensorial localizado na Faculdade de Ciências Agrárias, na Universidade Federal do Amazonas (UFAM). As amostras foram apresentadas em temperatura de refrigeração e os julgadores foram devidamente orientados para o preenchimento correto das fichas de avaliação.

Para a análise sensorial, foi aplicado o teste de aceitação adotando a escala hedônica com nove categorias com os extremos "gostei extremamente" e "desgostei extremamente" que avaliou o quanto os julgadores gostaram ou desgostaram das amostras segundo os atributos sensoriais de aparência, cor, aroma, sabor e textura (Ial, 2008).

Participaram da análise 50 provadores não treinados de ambos os sexos, com idade entre 18 e 49 anos, cada um recebeu a amostra em copo descartável de $50 \mathrm{~mL}$, a mesma foi codificada com três dígitos aleatórios. Na cabine, os provadores receberam a ficha de avaliação e foram feitas as devidas recomendações para não comprometer o método de análise.

\section{Resultados e Discussão}

Os resultados estão de acordo com a escala hedônica de nove pontos com os extremos "gostei extremamente" e “desgostei extremamente". Estão apresentados na Figura 1, onde há as médias correspondentes aos atributos sensoriais: aparência, cor, aroma, sabor e textura, respectivamente. Verifica-se que o atributo sabor obteve a maior nota média $(8,08)$, seguido pela cor $(7,54)$, a textura $(7,42)$, o aroma $(6,94)$ e a aparência $(6,9)$.

De acordo com a pesquisa desenvolvida por Pinke; Simoni; Pinto-e-Silva (2020), que avaliaram a influência dos aspectos sensoriais na escolha dos alimentos, o fator apelo sensorial, que corresponde à aparência, aroma, sabor e textura, foi citado como muito importante, além disso, o sentido sensorial que mais influenciou na escolha foi o sabor. Ressalta-se que 
quando o alimento possui uma qualidade sensorial e consegue mantê-la, contribui para a fidelidade do consumidor com o produto (Teixeira, 2009).

Na Figura 2, que consiste na aceitação global do iogurte, no atributo aroma, obteve-se que a maior nota foi 8 (gostei muito), com 36\%, entretanto as notas variaram de 9 a 7 pontos, totalizando $66 \%$ em aceitação. No iogurte grego desenvolvido por Demarco, et al. (2016), adicionado com diferentes doces típicos no oeste catarinense também encontraram um valor médio 8 para o iogurte grego com doce de morango $(8,20)$. Os autores Valadares, et al. (2017), também encontraram nota 8 na elaboração do iogurte grego com geleia de abacaxi.

Figura 1: Valores médios aos atributos sensoriais. Figura 2: Aceitação global do iogurte, no atributo aroma. Escala hedônica.
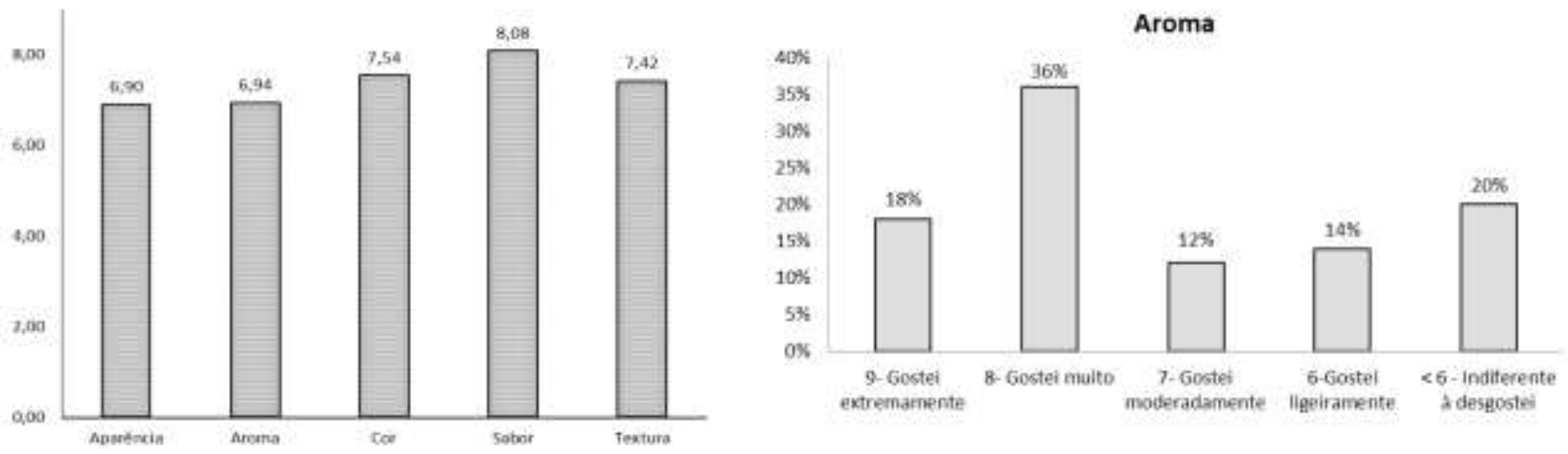

Fonte: Autores (2021).

Na Figura 3, apresenta-se o atributo sensorial aparência, cuja maior nota foi o 7 (gostei moderadamente), com 34\%, entretanto as notas variaram de 9 a 7 pontos, totalizando $68 \%$ em aceitação. O iogurte grego com geleia de goiabada enriquecido com inulina elaborado por Araújo, et al. (2018), também obteve maior média como "gostei moderadamente" nesse atributo.

De acordo com Lima, et al. (2011), a textura do iogurte é influenciada devido a dois fatores, a adição de sólidos e o tratamento, um exemplo de ingrediente que pode contribuir para aumentar a firmeza é o leite em pó integral ou desnatado. Na Figura 4, observa-se que o atributo sensorial textura obteve o 8 (gostei muito), com 32\%, como maior nota, entretanto as notas variaram de 9 a 7 pontos, totalizando 76\% em aceitação. Essa aceitação também foi encontrada por Demarco, et al. (2016), para a consistência do iogurte grego com doce de figo, abóbora, morango e pêssego. Assim como no iogurte grego com geleia de abacaxi de Valadares, et al. (2017). 
Figura 3: Aceitação global do iogurte, no atributo aparência.

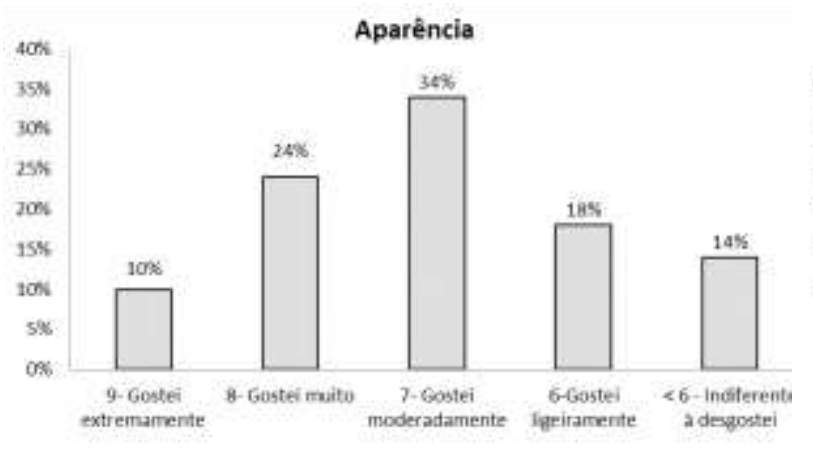

Figura 4: Aceitação global do iogurte, no atributo textura.

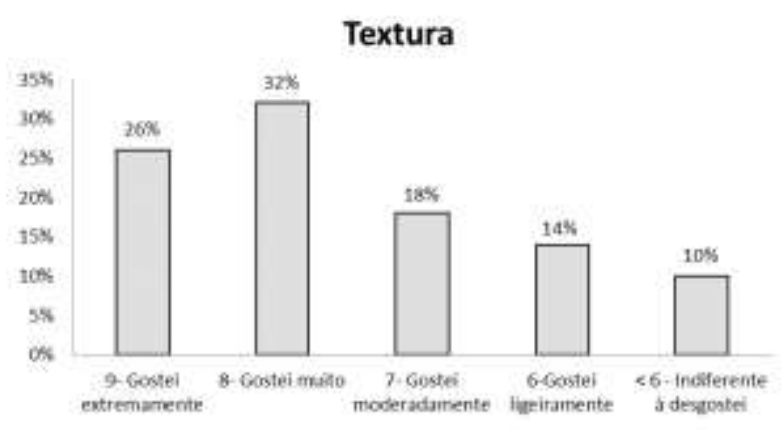

Fonte: Autores (2021).

A diferença entre os alimentos é identificada a partir do sabor e ele é influenciado por efeitos táteis, térmicos, dolorosos e/ou sinestésicos (Teixeira, et al., 2009). O atributo sensorial sabor que obteve a maior nota o 9 (gostei extremamente) com $42 \%$, entretanto as notas variaram de 9 a 7 pontos, totalizando $94 \%$ em aceitação satisfatória do produto (Figura 5). O iogurte grego com doce de abacaxi elaborado por Gomes, et al. (2006) nesse atributo foi o que obteve maior média em comparação aos outros iogurtes gregos produzidos com doces de frutas amazônicas.

E, por fim, na Figura 6 apresenta-se o atributo sensorial cor, que obteve a maior nota o 7 (gostei moderadamente) com $36 \%$, entretanto as notas variaram de 9 a 7 pontos, totalizando $88 \%$ em aceitação. A cor também foi obtida como gostei moderadamente por Demarco, et al. (2016), na elaboração de três tipos de iogurte grego com diferentes doces, o com doce de uva $(7,85)$, com doce de figo $(7,98)$ e com doce de pêssego $(7,89)$. Esse atributo é bastante importante, visto que é o primeiro contato que o consumidor tem com um tipo de produto, a cor em conjunto com a aparência que torna o produto ser aceito ou rejeitado (Teixeira, 2009).

Figura 5: Aceitação global do iogurte, no atributo Figura 6: Aceitação global do iogurte, no atributo cor. sabor.
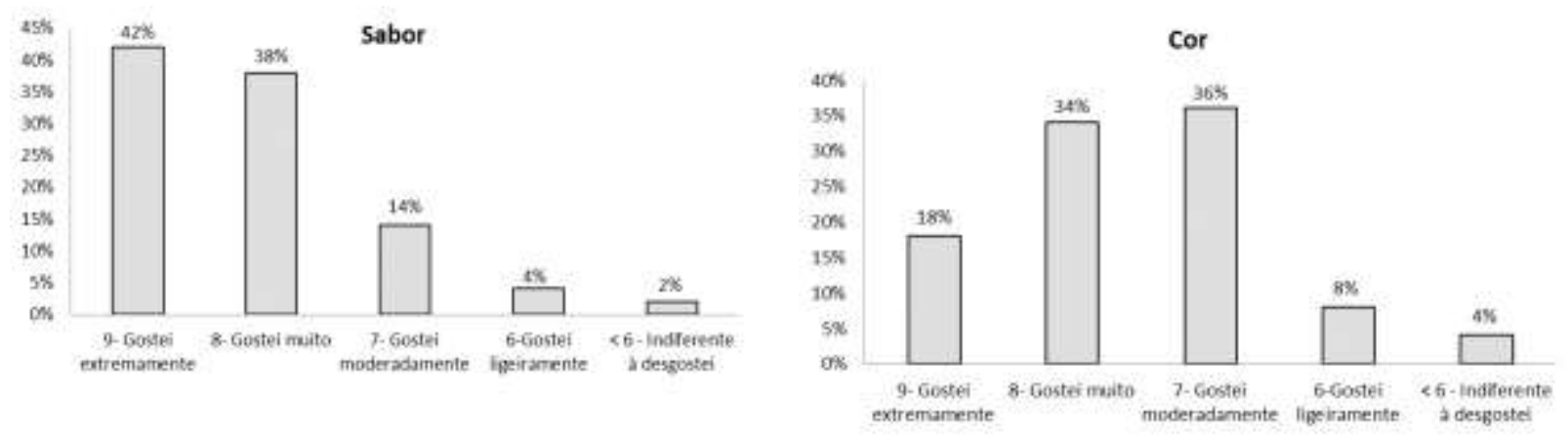

Fonte: Autores (2021).

Portanto, de modo geral é possível observar que o iogurte tipo grego com adição de doce de buriti foi bastante apreciado e aceito pelos provadores levando em consideração os atributos avaliados. 


\section{Conclusão}

Conclui-se que o iogurte tipo grego com adição de doce de buriti pode ser considerado uma ótima opção para o consumo de derivados do leite e recomendado como uma ideia inovadora no mercado de iogurtes, principalmente pela adição de sabor de fruta da Amazônia, dessa forma dando uma identidade regional para o produto.

Sugere-se a realização de estudos mais aprofundados, enfatizando a potencialidade da fruta amazônica para a produção de produtos alimentícios que possam agregar valor comercial à mesma, analisando a intenção de compra dos consumidores e caracterização do produto (como estudo de vida de prateleira e análise de antioxidantes, por exemplo). Desse modo, é possível obter mais informações e identificar maiores benefícios a partir do consumo do iogurte grego com doce de buriti.

\section{Agradecimentos}

À professora Dra. Eyde Cristianne Saraiva Bonatto da Universidade Federal do Amazonas pela orientação na condução desta pesquisa.

\section{Referências}

Aguiar, J. P. L., \& Souza, F. C. A. (2017). Desidratação e pulverização de polpa de buriti (Mauritia flexuosa L.): avaliação da vida de prateleira. Revista Brasileira de Fruticultura, 39, 1-7.

Araujo, P. V. et al. (2018). Elaboração e aceitação sensorial de iogurte grego com geleia de goiaba enriquecido com inulina. In: IV Encontro Nacional da Agroindústria. João Pessoa. Anais eletrônicos... Campinas, Galoá. https://proceedings.science/enag/enag-2018/papers/elaboracao-e-aceitacao-sensorial-deiogurte-grego-com-geleia-de-goiaba-enriquecido-com-inulina

Aryana, K. J., \& Olson, D. W. (2017). Uma revisão de 100 anos: Iogurte e outros produtos lácteos cultivados. Journal of Dairy Science, 100(12), 998710013.

Azoubel, P. M., \& Barros e Silva, S. A. (2007). Iogurte Saborizado com Polpa de Umbu. Embrapa. https://ainfo.cnptia.embrapa.br/digital /bitstream/C PATSA/35968/1/INT81.pdf

Beltrão, N. E. M., \& Oliveira, M. I. P. (2007). Oleaginosas Potenciais do Nordeste para a Produção de Biodiesel. Campina Grande: Embrapa Algodão.

Bezerra, K. C. A. et al. (2019). Perfil físico-químico e sensorial de iogurtes grego naturais elaborados com diferentes concentrações de sacarose. Revista Engenharia na Agricultura - Reveng, 27(2), 89-97, 2019. 10.13083/reveng.v27i2.832

Brasil. (2007). Instrução Normativa ${ }^{\circ} 46$, de 23 de novembro de 2007. Regulamento Técnico de Identidade e Qualidade (PIQ) de Leites Fermentados. Ministério da Agricultura, Pecuária e Abastecimento (MAPA). https:/www.diariodasleis.com.br/legislacao/federal/179713-regulamento-tucnico-leitesfermentados-adota-o-regulamento-tucnico-de-identidade-e-qualidade-de-leites-fermentados-anexo-u-presente-instruuuo-normativa.html

Brasil. (2017). Ministério da Agricultura e do Abastecimento - MAPA. Resolução no 5, de 13 de novembro de 2000, de Departamento de Inspeção de Produtos de Origem Animal. http://www.cidasc.sc.gov.br/inspecao/files/2012/08/RESOLU\%C3\%87\%C3\%83O-07_00_funcionamento-queijarias.pdf

Oliveira e Silva, A. M. et al. (2012). In vivo and in vitro antioxidant activity and hepatoprotective properties of polyphenols from Halimeda opuntia (Linnaeus) Lamouroux. Redox Report, 17(2), 47-53. 10.1179/ 1351000212Y.0000000003

Castro, Deise S. et al. (2014). Caracterização física e físico-química de polpa de buriti (Mauritia flexuosa). Revista Verde, 9(2), 117 - 120.

Demarco, G. et al. (2016). Elaboração e análise sensorial de iogurte grego adicionado de doces de frutas típicos do oeste catarinense. In: XXV Congresso Brasileiro de Ciência Tecnologia de Alimentos. Gramado. Anais... UFRG. http://www.ufrgs.br/sbctars-eventos/xxvcbcta/anais/files/110.pdf

Gao, Y. et al. (2012). Fucoidan, a sulfated polysaccharide from brown algae, improves cognitive impairment induced by infusion of Abeta peptide in rats. Environmental Toxicology and Pharmacology, 33(2), 304-311.

Gava, A. J., Silva, C. A. B. Da, \& Frias, J. R. G. (2008). Tecnologia de alimentos: princípios e aplicações. Nobel.

Gomes, L. B. et.al. (2016). Aceitabilidade do iogurte tipo grego com adição de doces de frutas da Amazônia. In: Congresso Técnico Científico De Engenharia E Da Agronomia, Foz do Iguraçu. Anais eletrônicos... Foz do Iguaçu, Paraná.

IAL. (2008). Instituto Adolfo Lutz. Métodos químicos e físicos para análise de alimento. (4a ed.), Normas analíticas do Instituto Adolfo Lutz, v.1, p. 989.

Kailasapathy, K., \& Chin, J. (2000). Survival and therapeutic potential of probiotic organisms with reference to Lactobacillus acidophilus and Bifidobacterium spp. Immunology and Cell Biology, 78(1), 80-88. 10.1046 / j.1440-1711.2000.00886.x 
Lima, S. C. G. et al. (2011). Efeito da adição de diferentes sólidos na textura, sinérese e característica sensorial de iogurte firme. Rev. Inst. Latic. "Cândido Tostes", 383(66), 32-39.

Loureiro, M. N. et al. (2013). Armazenamento de buriti em pó: efeito da embalagem nas características físicas e químicas. Biosci. J., 29(5), 1092-1100.

Ndabikunze, B. K. et al. (2017). Development and sensory evaluation of yoghurt flavoured with solar dried fruits. Journal of Agricultural Science and Food Technology, 3(7), 125-131.

Pereira, A. S., Shitsuka, D. M., Perreira, F. J, Shitsuka R. (2018). Metodologia da pesquisa científica. UFSM. https://repositorio.ufsm.br/bitstream /handle/1/15824/Lic_Compu tacao_Metodologia-Pesquisa-Cientifica.pdf?sequence=1.

Pinke, J. B., Simoni, N. K., Pinto-e-silva, M. E. M. (2020). Influência dos aspectos sensoriais na escolha dos alimentos. Segurança Alimentar e Nutricional, Campinas, SP, v. 27, p. e020021, 2020. https://periodicos.sbu.unicamp.br/ojs/index.php/san/article/view/8657218

Teixeira, L. V. (2009). Análise sensorial na indústria de alimentos. Revista do Instituto de Laticínios Cândido Tostes, 64(366), 12-21.

Uduwerella, G., Chandrapala, J., \& Vasiljevic, T. (2017). Minimising generation of acid whey during Greek yoghurt manufacturing. Journal of Dairy Research, 84(3), 346-354.

Valadares, P. L. O. et al. (2017). Desenvolvimento do iogurte grego com geleia de abacaxi: análise sensorial e intenção de consumo. In: VIII Jornada de Iniciação Científica e Extensão, 8. Tocantins. Anais eletrônicos.... Palmas. https://propi.ifto.edu.br/ocs/index.php/jice/8jice/paper/viewFile/8507/3889

Vieira, R. F. et al. (2010). Frutas nativas da região Centro-oeste do Brasil. Embrapa Informações Tecnológica. 322.

Wang, J. et al. (2012). Effect and mechanism of fucoidan derivatives from Laminaria japonica in experimental adenine-induced chronic kidney disease. Journal of Ethnopharmacology, 139(3), 807-813. 